\title{
A3 钢在芽孢杆菌作用下的腐蚀行为
}

\author{
杜 娟 李松梅* 刘建华 于 美 \\ (北京航空航天大学材料科学与工程学院, 空天材料与服役教育部重点实验室, 北京 100191)
}

\begin{abstract}
摘要：采用表面分析技术、失重法和电化学测试技术研究了 A3 钢在芽狍杆菌(Bacillus)作用下的腐蚀行为. 扫 描电子显微镜(SEM)分析结果表明, 浸泡 $7 \mathrm{~d}$ 时, 芽狍杆菌会在 A3 钢表面形成致密的生物膜, 有效阻隔了溶液对 基体的腐蚀, 抑制了腐蚀过程. 电化学交流阻抗测试结果显示, 含菌体系中的试样表面经历了 2 个时间常数 $\rightarrow 3$ 个时间常数 $\rightarrow 2$ 个时间常数的变化过程. 失重法和极化曲线测试结果表明, 芽狍杆菌的细菌活性对生物膜的保 护作用有着决定性的影响, 当微生物活性下降, 生物膜的保护能力也会大幅降低.
\end{abstract}

关键词：电化学交流阻抗谱; 芽狍杆菌; 微生物腐蚀; 表面分析技术; 失重法; 极化曲线 中图分类号：0646

\section{Corrosion Behavior of Steel A3 Influenced by Bacillus}

\author{
DU Juan LI Song-Mei * LIU Jian-Hua YU Mei
}

(Key Laboratory of Aerospace Materials and Performance, Ministry of Education, School of Materials Science and Engineering, Beihang University, Beijing 100191, P. R. China)

\begin{abstract}
Surface analysis, mass loss and electrochemical mesurements were used to investigate the corrosion behavior of steel A3 under the effect of Bacillus. Scanning electron microscopy (SEM) indicated that the presence of Bacillus resulted in a compact biofilm after 7 days of exposure, which effectively protected the coupon from being corroded by the solution. Electrochemical impedance spectroscopy (EIS) showed that the number of time constants changed from 2 to 3 and returned to 2 in the Bacillus system. The mass loss and polarization curve results showed that the activity of the bacteria determined the effectiveness of the biofilm. The protective ability of the biofilm decreased when the activity of the bacteria decreased.
\end{abstract}

Key Words : Electrochemical impedance spectroscopy; Bacillus; Microbiologically influenced corrosion; Surface analysis method; Weight loss method; Polarization curve

微生物腐蚀(microbiology influenced corrosion, 简称 MIC) 是指由于生物膜内微生物的存在和其生 命活动而引起的腐蚀现象 ${ }^{[1]}$. 研究表明, 在水、湿润 空气等有利于细菌存在和生长的场所, 如发电厂冷 却水循环系统、热交换系统, 石油开采、储存和运输 系统、污水处理管道, 饮用水管道, 飞机燃油储存罐 等都有不同程度的微生物浸染及其造成的腐蚀 ${ }^{[2]}$. 目前, 关于微生物腐蚀方面的报道多集中于硫酸盐 还原菌 $(\mathrm{SRB})$ 对不同材料的腐蚀行为 ${ }^{[3-7]}$, 然而在实 际工作环境中, 部分好气性细菌及真菌也会对腐蚀
过程起到一定作用 ${ }^{[8]}$.

芽孢杆菌(Bacillus) 是一种好气性异养菌, 利用 多种有机物进行严格呼吸代谢, 严格发酵代谢或呼 吸和发酵二者兼有的代谢 ${ }^{[9]}$. 国内外关于芽孢杆菌 的研究与应用已有 100 多年的历史, 早期大部分工 作集中在形态观察、分类鉴定、生理机制、功能发掘 及防治等方面 ${ }^{[10]}$. 近年来, 人们逐渐转向了芽孢杆菌 在工业、农业、医药及生物学理论领域的应用研 究 ${ }^{[8,11-13]}$, 而关于芽狍杆菌引起的碳钢腐蚀行为的报 道却较少. 近年来, 我们在汽油储油罐的底部分离得

Received: November 5, 2009; Revised: March 10, 2010; Published on Web: April 16, 2010.

"Corresponding author. Email: songmei_li@buaa.edu.cn; Tel: +86-10-82317103 
到了芽孢杆菌, 并对芽孢杆菌对 A3 钢腐蚀行为的 影响进行了研究, 旨在为微生物腐蚀控制方法的提 出提供理论依据.

\section{1 材料与实验方法}

\section{1 实验材料}

实验所用材料为 $\mathrm{A} 3$ 钢, 其化学成分为: $\mathrm{C}(\leqslant$ $0.3 \%), \mathrm{Si}(\leqslant 0.01 \%), \mathrm{Mn}(\leqslant 0.42 \%), \mathrm{S}(\leqslant 0.029 \%), \mathrm{P}$ $(\leqslant 0.019 \%)$. 将材料分别加工成 $20 \mathrm{~mm} \times 30 \mathrm{~mm} \times 2$ $\mathrm{mm}$ (长方形)和 $10 \mathrm{~mm} \times 10 \mathrm{~mm} \times 2 \mathrm{~mm}$ (正方形)两种 方形带孔试片和 $\Phi 10 \mathrm{~mm} \times 10 \mathrm{~mm}$ 圆形试片. 第一 种长方形试片打磨至 1200 , 用于挂片腐蚀实验并 进行腐蚀形貌观察, 另外一种正方形试片打磨至 2000\# 后抛光, 用于生物膜观察; 圆形试片做成电 极, 用于电化学实验, 电极的工作面用水砂纸打磨至 1200\#. 所有试样均用无菌去离子水冲洗, $75 \%$ 酒精 消毒后置于无菌干燥箱中保存, 备用. 在实验前再用 紫外灯灭菌 $30 \mathrm{~min}$, 确保实验中无杂菌污染.

\section{2 菌种来源与培养}

实验菌种芽狍杆菌是从成品油的储油罐底部分 离并提纯得到的. 为了拟合实际环境, 培养基采用改 良后的 Starkey 培养基: $\mathrm{FeSO}_{4} 0.01 \mathrm{~g} \cdot \mathrm{L}^{-1}, \mathrm{CaCl}_{2} 0.25$ $\mathrm{g} \cdot \mathrm{L}^{-1}, \mathrm{MgSO}_{4} 0.5 \mathrm{~g} \cdot \mathrm{L}^{-1},\left(\mathrm{NH}_{4}\right)_{2} \mathrm{SO}_{4} 0.3 \mathrm{~g} \cdot \mathrm{L}^{-1}, \mathrm{KH}_{2} \mathrm{PO}_{4}$ $3.5 \mathrm{~g} \cdot \mathrm{L}^{-1}, \mathrm{~S} 5 \mathrm{~g} \cdot \mathrm{L}^{-1}$ 与马铃薯培养基(PDA)以 $2: 1$ 的 体积比混合构成混合培养基, $\mathrm{pH}$ 值为 $(4.0 \pm 0.1)$, 实 验中所有试剂均为分析纯. 按无菌操作方法将处于 休眠状态的芽孢杆菌二次活化后, 以 $10 \%$ 的比例接 种于新鲜的混合培养基中, 置于 $37{ }^{\circ} \mathrm{C}$ 恒温震荡培养 箱(GNP-9270 型, 上海精宏实验设备有限公司)中进 行培养. 微生物的计数采用稀释平板计数法测得溶 液中的活菌数量进而绘制出细菌的生长曲线, 实验
采用固体马軨薯琼脂培养基对微生物进行培养.

\section{3 生物膜分析}

将 A3 钢方形试片打磨至 2000 , 用 $0.5 \mu \mathrm{m}$ 的 研磨膏抛光, 丙酮去油, 用紫外灯灭菌后浸泡在含有 芽孢杆菌的腐蚀体系中, $7 \mathrm{~d}$ 后取出, 干燥, 然后用扫 描电镜(JSM-5800 型, 日本电子造)观察生物膜形貌, 并利用傅里叶变换红外光谱仪(360FT-IR ESP 型, 美国 Nicolet 公司造)对生物膜成分进行分析.

\section{4 电化学测试}

电化学测试采用仪器为美国普林斯顿公司生产 的 PAR2273 电化学工作站. 工作电极为 A3 钢, 参 比电极为饱和甘录电极, 辅助电极为大面积铂电极. 测量工作电极在空白培养基中和有菌体系中浸泡 $0 、 2 、 7 、 14 、 21 \mathrm{~d}$ 的电化学阻抗谱(EIS)及极化曲线. EIS 在自腐蚀电位下进行测试, 激励信号为 $5 \mathrm{mV}$ 的 正弦波, 测试频率范围为 $10 \mathrm{mHz}-100 \mathrm{kHz}$. Tafel 极 化曲线的测定采用动电位扫描方式, 初始电位负于 开路电位 $0.15 \mathrm{~V}$, 结束电位正于开路电位 $0.25 \mathrm{~V}$, 电 位扫描速率为 $0.5 \mathrm{mV} \cdot \mathrm{s}^{-1}$.

\section{5 腐蚀产物形貌及失重分析}

将已灭菌的带孔长方形 A3 钢片分别浸在刚刚 接种微生物的培养基和空白培养基中, 每组 3 个平 行试样, $21 \mathrm{~d}$ 后将其从腐蚀介质中取出, 用扫描电子 显微镜观察腐蚀产物的形貌, 彻底去除腐蚀产物后 的试样用失重法计算平均腐蚀速率.

\section{2 结果与讨论}

\section{1 微生物的生长}

图 1 为芽狍杆菌的菌落形态及其接人混合培养 基后的生长曲线. 由图 1(a)可以看出, 芽孢杆菌的菌 落主要特征是表面粗糙、不透明、禇皱、乳白色, 基本
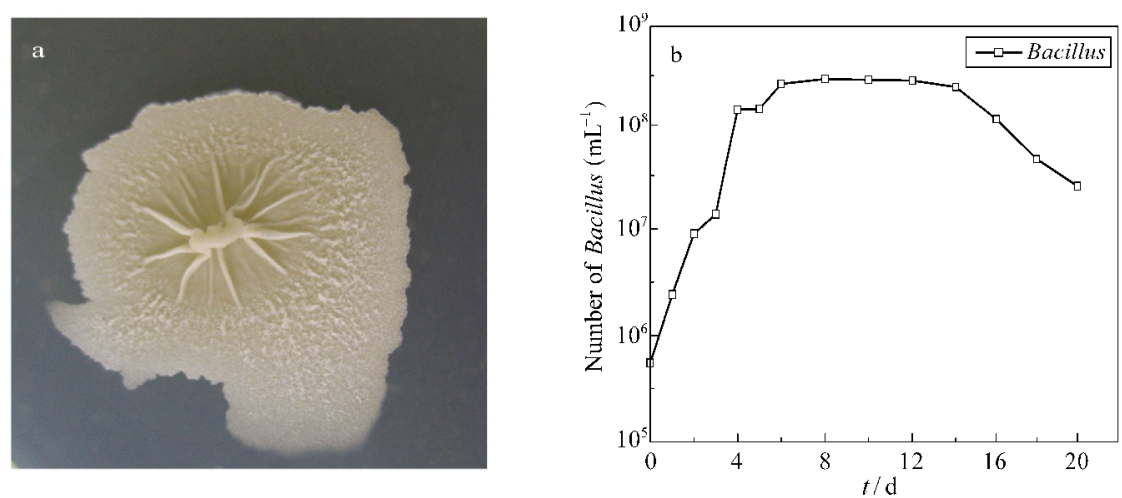

图 1 细菌菌落形态(a)及其在液体培养基中的生长曲线(b)

Fig.1 Morphologies of bacterium (a) and the growth curves of Bacillus (b) in the medium 
呈圆形, 边缘为裙边状, 中间有疤状突起. 菌落与培 养基的连接比较紧密, 用接种针不易挑起. 由图 l(b) 可知, 芽孢杆菌在接人培养基后很快就进入了快速 生长期, 并在第 $7 \mathrm{~d}$ 就达到了最大值, 这段时间内细 菌的新陈代谢最为旺盛、活性最大. 在第 $7 \mathrm{~d}$ 到 $14 \mathrm{~d}$, 微生物生长进人稳定期, 这段时间微生物的活性开 始下降, 只是维持数量基本不变, 直至第 $14 \mathrm{~d}$, 细菌 数量开始减少, 活性大幅度下降.

\section{2 生物膜形貌观察与成分分析}

当材料浸泡于含菌溶液时, 微生物会逐渐吸附 于材料表面, 并很快就会分泌胞外聚合物(extracelular polymer substances, EPS), 这层分泌物将微生物 牢牢地固定在材料表面, 完成了微生物从可逆吸附 向不可逆吸附的转变 ${ }^{[14]}$. 由此生成的生物膜在经历 了游离态到固着态转变过程的同时, 会生成特殊的 次级代谢产物, 并会引起生物、化学以及物理侵蚀等 方面阻抗值的显著增加 ${ }^{[15]}$. 图 $2 \mathrm{a}$ 为 A3 钢在芽孢杆 菌菌液中浸泡 $7 \mathrm{~d}$ 后形成的生物膜形貌. 从图中可 以看出, 试样表面包覆了一层由大小不一的杆状细 菌、胞外聚合物以及腐蚀产物相互粘结而成的复杂 的生物膜. 虽然该膜层上细菌分布并不十分均匀, 但 膜层相对致密, 对于基体有一定的保护作用.

图 $2 \mathrm{~b}$ 为生物膜的傅里叶变换红外光谱图, 图中 $3421 \mathrm{~cm}^{-1}$ 处的吸收峰与一 $\mathrm{OH}$ 伸缩振动有关; 1632 $\mathrm{cm}^{-1}$ 处吸收峰是 $-\mathrm{C}=\mathrm{C}$ 基团所特有的; $2927 \mathrm{~cm}^{-1}$ 左右出现的特征峰是由亚甲基- $\mathrm{C}-\mathrm{H}$ 基团的不 对称伸缩振动形成的; $1384 \mathrm{~cm}^{-1}$ 处的吸收峰为甲 基- $\mathrm{C}-\mathrm{H}$ 基团的对称弯曲振动; $1047 \mathrm{~cm}^{-1}$ 附近的 谱峰很可能是由亚甲基- $\mathrm{C}-\mathrm{H}$ 基团的耦合摆动造 成的. 胞外聚合物通过不同官能团间的相互作用, 帮 助细菌细胞和腐蚀产物相互黏结, 最终在试样表面
形成了较为致密、均匀的生物膜层, 有效地阻隔了溶 液中活泼离子(如 $\mathrm{SO}_{4}^{2-} 、 \mathrm{Cl}^{-}$等)对基体的侵蚀.

\section{3 交流阻抗测试}

微生物对腐蚀速率的影响可以用极化电阻 $\left(R_{\mathrm{p}}\right)$ 来衡量, 其中 $R_{\mathrm{p}}$ 与腐蚀电流密度 $\left(j_{\text {corr }}\right)$ 是呈反比的. $R_{\mathrm{p}}$ 值既可以从阻抗谱的低频区 $\left(Z_{\omega} \rightarrow 0\right)$ 读得, 也可以通 过对实验数据进行等效电路拟合来得到 ${ }^{[16]}$. 图 3 为 A3 钢分别浸泡在两个体系中 $0 、 2 、 7 、 14$ 及 $21 \mathrm{~d}$ 的 电化学阻抗图谱. 从图 $3\left(a_{1}, a_{2}, a_{3}\right)$ 可以看出, 浸泡于 空白培养基中的试样阻抗值经历了先增大后减小的 趋势, 第 $2 \mathrm{~d}$ 就达到最大值, 并且 $2 、 7$ 和 $14 \mathrm{~d}$ 的阻抗 值相差并不大. 前期阻抗值的增加可能与表面膜层 的累积有效阻隔了活泼离子对基体的侵蚀有关, 而 后期的减小是由于膜层脱落, 试样再一次暴露于培 养基中引起的. 其中通过图 $3 \mathrm{a}_{2}$ 可以看出, 在浸泡的 $0 、 2 、 7 、 14 \mathrm{~d}$ 的相图只存在 2 个时间常数(这说明表 面只存在一个明显的膜层结构); 而到第 $21 \mathrm{~d}$, 相图 出现了第 3 个时间常数, 这可能是由于空白培养基 中的试样表面膜层是以层为单位逐渐累积的, 当浸 泡至 $21 \mathrm{~d}$ 时, 膜层间结合力已经不足以维持成一个 整体, 使得膜层分裂为两个明显的独立膜层引起的. 从图 $3\left(b_{1}, b_{2}, b_{3}\right)$ 可以看出, 在芽孢杆菌中浸泡的试 样阻抗也经历了先增大后减小的过程, 不同的是: (1) 第 $2 \mathrm{~d}$ 的 Nyquist 图有感抗的存在, 这与微生物 的吸附、脱附及生物膜的逐渐形成有关; (2) 膜层的 阻抗不同于空白培养基, 是在第 $7 \mathrm{~d}$ 达到最大的, 这 与微生物的活性及膜层结构有着很大关系. 比较不 同浸泡时间下的相图(图 $3 b_{2}$ ) 可以看出, 前期 $(0 \mathrm{~d} 、 2$ d) 只存在两个时间常数, 这是生物膜开始发展的阶 段; 到浸泡的中期 $(7 \mathrm{~d} 、 14 \mathrm{~d})$ 出现了 3 个时间常数, 试样表面除了生物膜, 还有腐蚀产物膜逐渐形成, 此
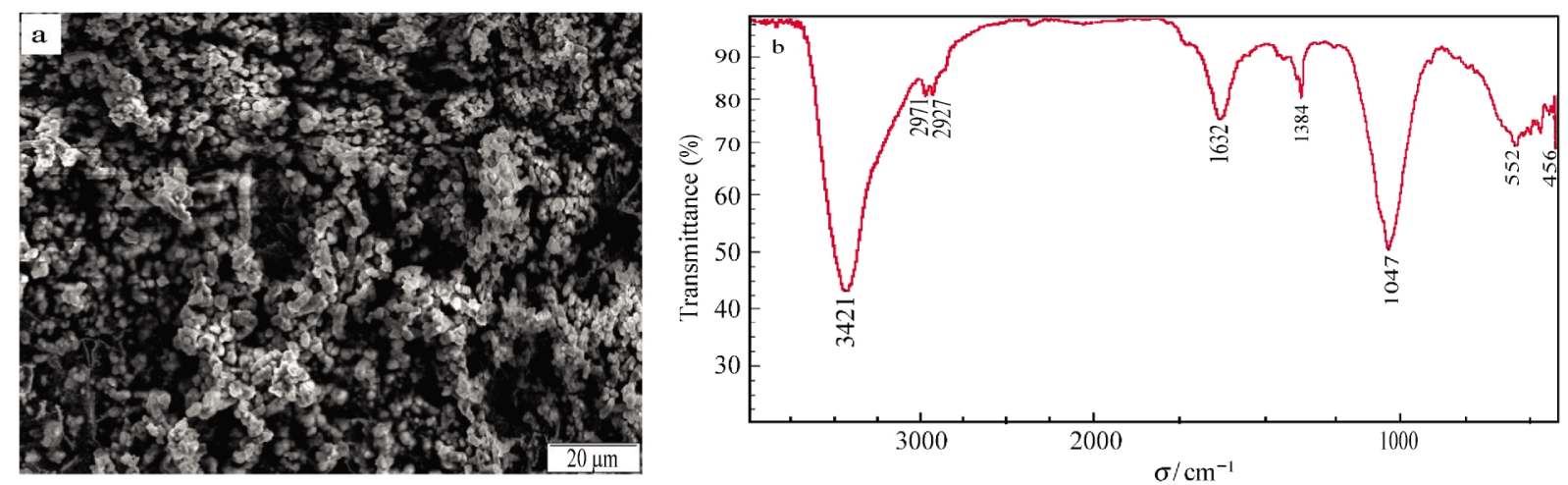

图 2 浸泡在芽孢杆菌菌液中 $7 \mathbf{d}$ 后 $\mathbf{A 3}$ 钢表面生物膜的形貌图(a)及傅里叶变换红外光谱图(b)

Fig.2 SEM image (a) and Fourier transform infrared spectrum (b) of the biofilms of steel A3 exposed in Bacillus solution for 7 days 

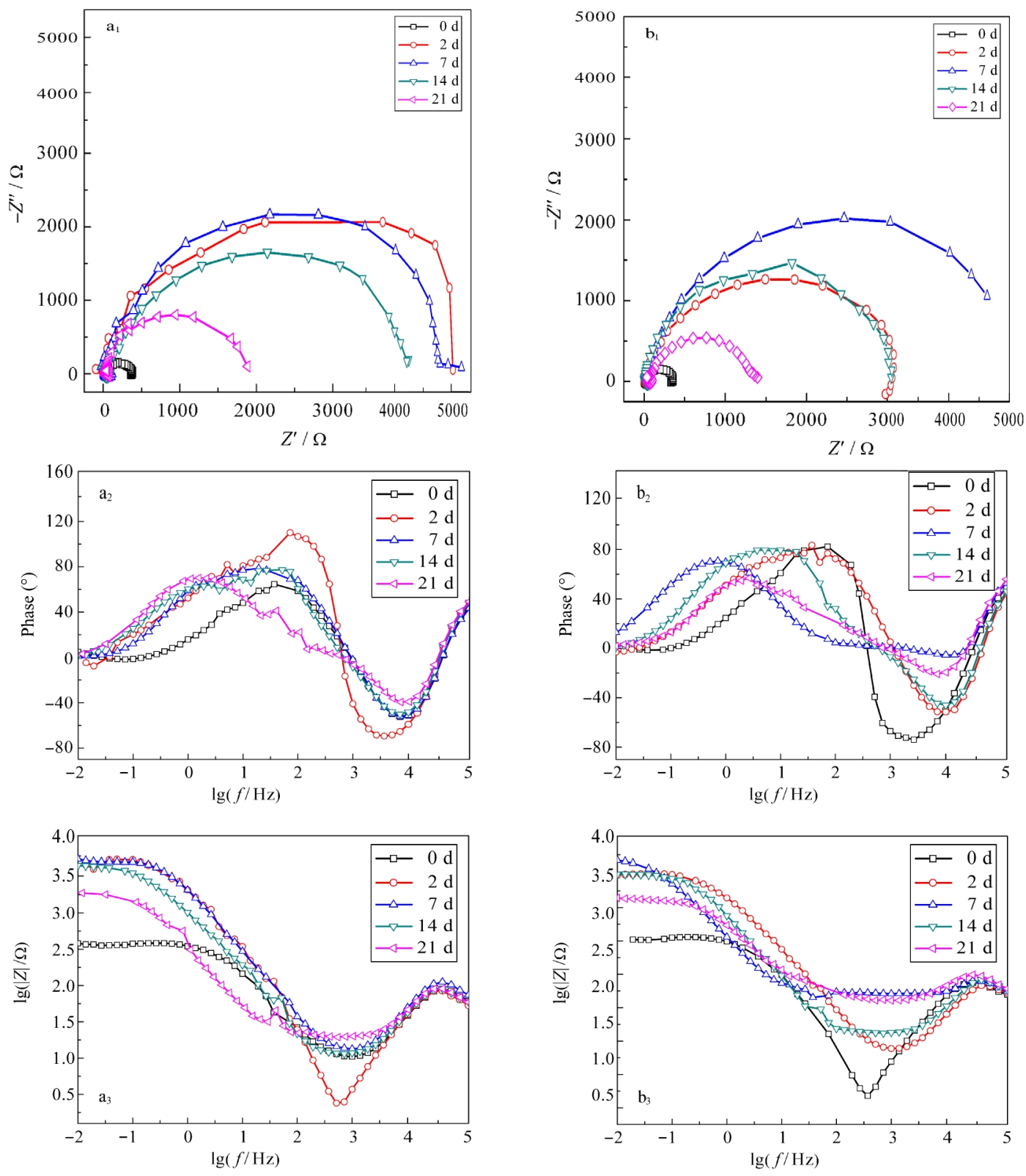

图 $3 A 3$ 钢浸泡在空白培养基 $\left(a_{1}, a_{2}, a_{3}\right)$ 和芽孢杆菌菌液 $\left(b_{1}, b_{2}, b_{3}\right)$ 中不同天数的电化学阻抗谱

Fig.3 EIS diagrams of steel $A 3$ after different days of exposure in sterile solution $\left(a_{1}, a_{2}, a_{3}\right)$ and Bacillus solution $\left(\mathbf{b}_{1}, \mathbf{b}_{2}, \mathbf{b}_{3}\right)$

时试样表面得到了较好的保护, 阻抗值达到最大; 当浸泡进行到第 $21 \mathrm{~d}$, 此时时间常数又变为 2 个, 这 是由于生物膜对基体的保护作用主要是在细菌具有 活性时才能发挥 ${ }^{[17]}$, 而此时微生物已基本死亡, 残余 的生物膜与腐蚀产物膜杂合形成的复杂膜层疏松而 多孔, 因此导致阻抗值的大幅减小. 对比不同体系的 阻抗谱会发现, 虽然空白培养基中试样的阻抗值在 很多测试时间大于含菌体系, 但是在微生物具有最 大活性时 $(7 \mathrm{~d})$, 含菌体系中试样的阻抗值大于无菌 体系, 此时完整的生物膜层以及 EPS 有效填补了腐
蚀产物间的空隙, 使得此时的膜层具有很强的保护 作用. 之后由于微生物活性下降, 生物膜对基体的保 护作用逐渐衰减, 含菌体系的阻抗值逐渐降低.

为了进一步阐述膜层结构对腐蚀过程的影响, 我们采用 ZSIMPWIN 软件对芽狍杆菌菌液中电极 典型特征的阻抗谱 $(2 、 7 、 14$ 及 $21 \mathrm{~d})$ 进行拟合, 各体 系的等效电路如图 4 所示. 由于电极在菌液中浸泡 后, 其粗糙的微观表面引起扩散作用的缘故 ${ }^{[18]}$, 电化 学测试所得膜层电容用常相位角原件 $Q$ 来拟合. 图 $4 \mathrm{a}$ 表明在浸泡 $2 \mathrm{~d}$ 的试样表面存在一个不够完整的 

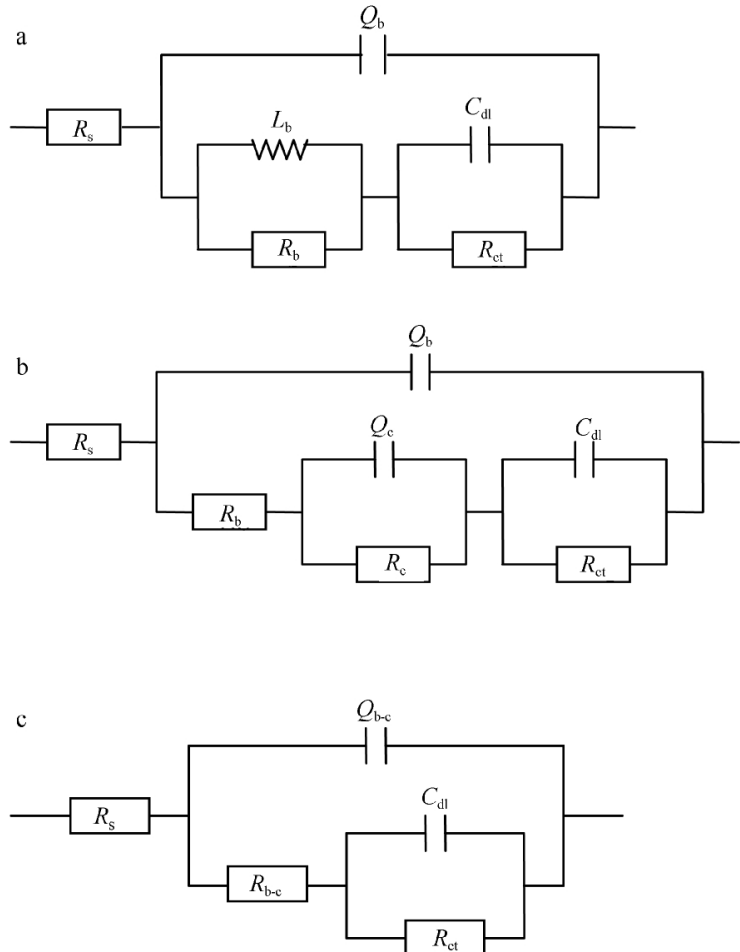
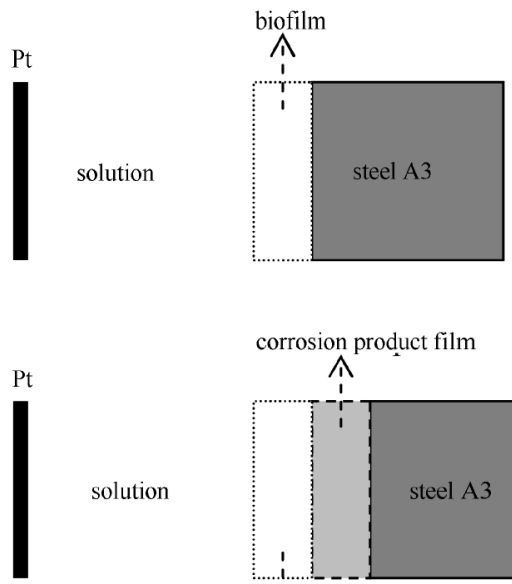

corrosion product film

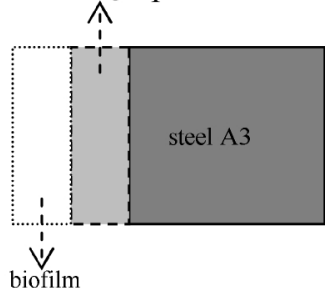

biofilm-product film
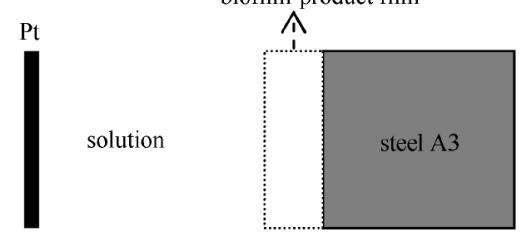

图 4 A3 钢在芽狍杆菌菌液中浸泡不同天数的等效电路图

Fig.4 Equivalent circuit models of steel A3 after different days of exposure in Bacillus solution

(a) model for electrodes in Bacillus solution for 2 days, (b) model for electrodes in Bacillus solution for 7 and 14 days, (c) model for electrodes in Bacillus solution for 21 days; The right figures are the corresponding schematics of electrodes with different films in Bacillus solution. $R_{\mathrm{s}}$ : solution resistance; $Q_{\mathrm{b}}$ : capacitance of the biofilm; $R_{\mathrm{b}}$ : resistance of the biofilm; $L_{\mathrm{b}}$ : inductance induced by the adhesion of the biofilm; $C_{\mathrm{dl} l}$ capacitance of the double layer; $R_{\mathrm{d}}$ : charge transfer resistance; $Q_{\mathrm{bcc}}$ c capacitance of the complex film formed by Bacillus and corrosion products; $R_{\mathrm{bc}}$ : resistance of the complex film formed by Bacillus and corrosion products; $Q_{\mathrm{c}}$ : capacitance of the corrosion products;

$R_{\mathrm{c}}$ : resistance of the corrosion products

生物膜将电极与溶液隔开; 图 $4 \mathrm{~b}$ 表明在浸泡的 7 、 $14 \mathrm{~d}$, 试样表面有两个膜层, 外层为生物膜, 内层为 腐蚀产物膜, 两个膜层共同保护基体, 减小溶液对金 属的溶解; 图 4c 表明浸泡的第 $21 \mathrm{~d}$, 试样表面存在 一个生物膜-产物膜共同组成的膜层. 图中所有元件 的拟合值列于表 1 .

比较表 1 中不同天数的 $Q_{\mathrm{b}}$ 及 $R_{\mathrm{b}}$ 值, 第 $2 \mathrm{~d}$ 与 第 $7 \mathrm{~d}$ 的电容值很相近 (即膜厚相当), 而电阻值增大 了 3 个数量级, 这说明在 $2-7 \mathrm{~d}$, 生物膜主要是通过 致密化引起电阻的增加; 到第 $14 \mathrm{~d}, Q_{\mathrm{b}}$ 值增大, 即生 物膜的厚度开始减小, 同时阻抗值也减小到 1705
$\Omega \cdot \mathrm{cm}^{2}$, 生物膜对基体的保护作用大幅降低. 比较不 同天数的 $Q_{\mathrm{c}}$ 及 $R_{\mathrm{c}}$ 值, $Q_{\mathrm{c}}$ 值的升高, 即膜厚的减小, 反而引起了阻抗值 $R_{\mathrm{c}}$ 的增加, 这是腐蚀产物层逐渐 致密的结果. 比较 7 和 $14 \mathrm{~d}$ 的 $R_{\mathrm{b}}$ 和 $R_{\mathrm{c}}$ 值可以看出, 对于基体的保护, 生物膜是起主导作用的. 而到了第 $21 \mathrm{~d}$, 表面膜层以腐蚀产物为主, 剩余生物膜掺杂其 中, 此时的膜层阻抗值降低到只有 $212.6 \Omega \cdot \mathrm{cm}^{2}$, 甚 至低于 $14 \mathrm{~d}$ 的 $R_{\mathrm{c}}$ 值 $\left(405.5 \Omega \cdot \mathrm{cm}^{2}\right)$ ，这可能是由于微 生物大量存在时产生的 EPS 渗人到腐蚀产物间, 填 补了产物间的缝隙, 使得腐蚀产物膜具有一定的机 械阻隔能力, 随着细菌的死亡, EPS 的溶解, 这种保

表 1 拟合电路中元件的拟合值

Table 1 Parameter values for each element in the equivalent circuit model

\begin{tabular}{ccccccccccccc}
\hline \multirow{2}{*}{ System } & \multirow{2}{*}{$t / \mathrm{d}$} & $\frac{R_{\mathrm{s}}}{n}$ & $\frac{10^{5} Q_{\mathrm{b}}}{\left(\Omega \cdot \mathrm{cm}^{2}\right)}$ & $\frac{R_{\mathrm{b}}}{\left(\mu \mathrm{F} \cdot \mathrm{cm}^{-2}\right)}$ & $\frac{1}{\left(\Omega \cdot \mathrm{cm}^{2}\right)}$ & $10^{4} L_{\mathrm{b}} / \mathrm{H}$ & $\frac{10^{5} Q_{\mathrm{bc}}}{\left(\mu \mathrm{F} \cdot \mathrm{cm}^{-2}\right)}$ & $\frac{R_{\mathrm{bc}}}{\left(\Omega \cdot \mathrm{cm}^{2}\right)}$ & $\frac{10^{5} Q_{\mathrm{c}}}{\left(\mu \mathrm{F} \cdot \mathrm{cm}^{-2}\right)}$ & $\frac{R_{\mathrm{c}}}{\left(\Omega \cdot \mathrm{cm}^{2}\right)}$ & $\frac{10^{5} C_{\mathrm{dl}}}{\left(\mu \mathrm{F} \cdot \mathrm{cm}^{-2}\right)}$ & $\frac{R_{\mathrm{ct}}}{\left(\Omega \cdot \mathrm{cm}^{2}\right)}$ \\
\hline Bacillus & 2 & 6.802 & 8.100 & 7.929 & 5.61 & - & - & - & - & 4.440 \\
& 7 & 52.31 & 8.446 & 3878 & & & & & & & & 3207 \\
& 14 & 13.67 & 18.23 & 1705 & - & - & - & 266.7 & 405.5 & 19.17 & 940.4 \\
& 21 & 40.82 & - & - & - & 23.39 & 212.6 & - & - & 11.90 & 1090 \\
\hline
\end{tabular}




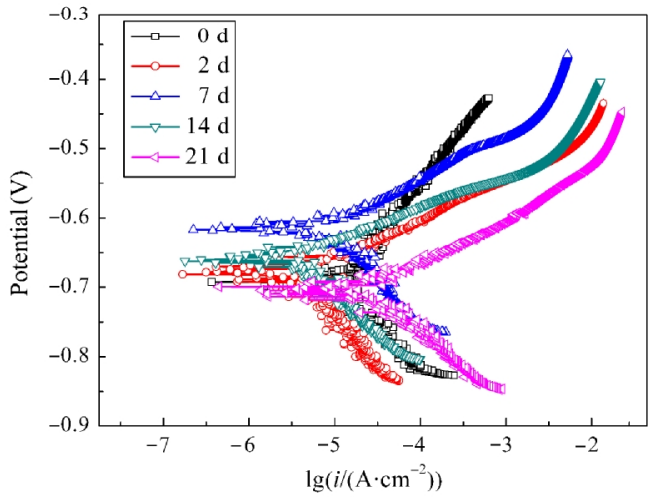

图 $5 \mathrm{~A} 3$ 钢浸泡在芽狍杆菌菌液中不同天数的极化曲线

Fig.5 Polarization curves of steel $\mathrm{A} 3$ after different days of exposure in Bacillus solution

护能力也随之下降.

\subsection{Tafel 极化曲线测试}

图 5 为 $\mathrm{A} 3$ 钢在芽孢杆菌菌液中浸泡不同天数 所得的极化曲线. 从极化曲线可以看出, 细菌的存在 引起了曲线阳极极化部分的波动, 其中细菌活性最 大的 $7 \mathrm{~d}$ 引起的变化也最大, 这可能是由于细菌的 生命活动影响到了阳极反应过程. 比较不同天数的 自腐蚀电位, 可以看出, 在 0-21 d 的浸泡过程中, 电 位经历了先正移 $(7 \mathrm{~d}$ 达到最正)后负移 $(21 \mathrm{~d}$ 达到最
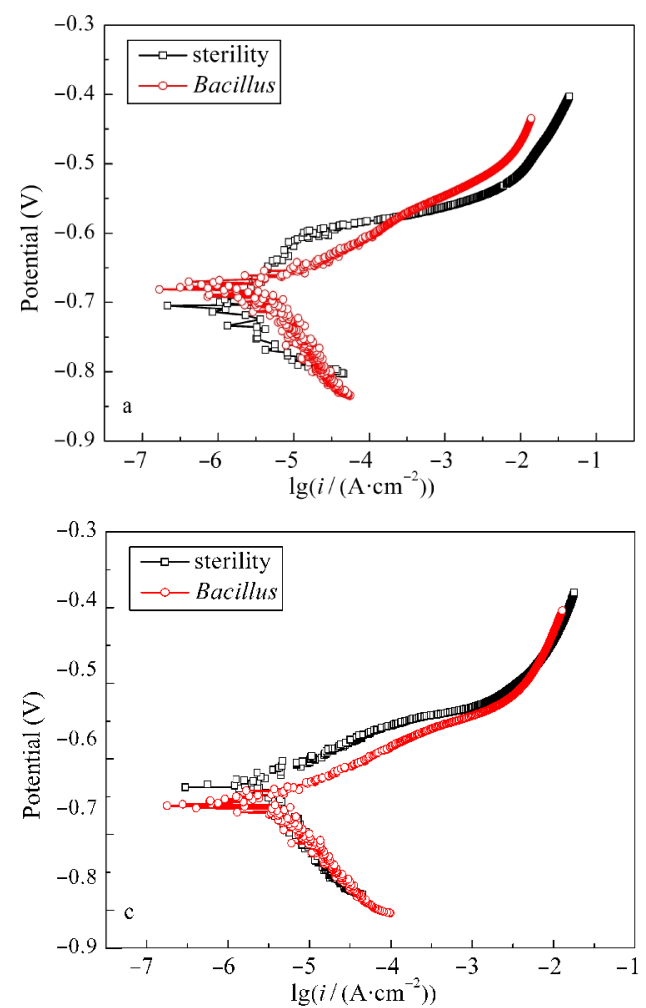

负)的过程, 即 A3 钢电极的腐蚀倾向在细菌活性最 高时降到最低, 随着细菌的逐渐死亡, 腐蚀倾向也逐 渐增大. 第 $21 \mathrm{~d}$ 的自腐蚀电流密度最大, 这与失重 测试及交流阻抗测试所得结论一致, 即 0-21 d 内, 此时 A3 钢的腐蚀速率是最大的.

图 6 为 $\mathrm{A} 3$ 钢电极在不同体系中分别浸泡 $2 、 7$ 、 14 和 $21 \mathrm{~d}$ 后极化曲线的对比, 表 2 为根据极化曲线 测得的自腐蚀电流密度值. 可以看出, 在第 2-7 d 时,含菌体系的自腐蚀电位均正于空白培养基体系, 此时高活性微生物作用下的 A3 钢电极表面具有较 小的腐蚀倾向. 在完整致密的生物膜包覆下, 电极自 腐蚀电流密度较小, 腐蚀速率较低. 当浸泡时间达到 $14 \mathrm{~d}$ 时, 含菌体系中电极的自腐蚀电位开始低于空

表 2 A3 钢电极浸泡在无菌溶液和芽狍杆菌菌液中不同天 数的自腐蚀电流密度

Table 2 Corrosion current densities of steel A3 electrodes exposed in sterile and Bacillus solutions for different days

\begin{tabular}{ccccc}
\hline \multirow{2}{*}{ System } & \multicolumn{4}{c}{$\lg \left(i /\left(\mathrm{A} \cdot \mathrm{cm}^{-2}\right)\right)$} \\
\cline { 2 - 5 } & $2 \mathrm{~d}$ & $7 \mathrm{~d}$ & $14 \mathrm{~d}$ & $21 \mathrm{~d}$ \\
\hline sterility & -5.8059 & -5.5702 & -5.5592 & -5.2586 \\
Bacillus & -5.3442 & -4.9551 & -5.4348 & -4.6263 \\
\hline
\end{tabular}
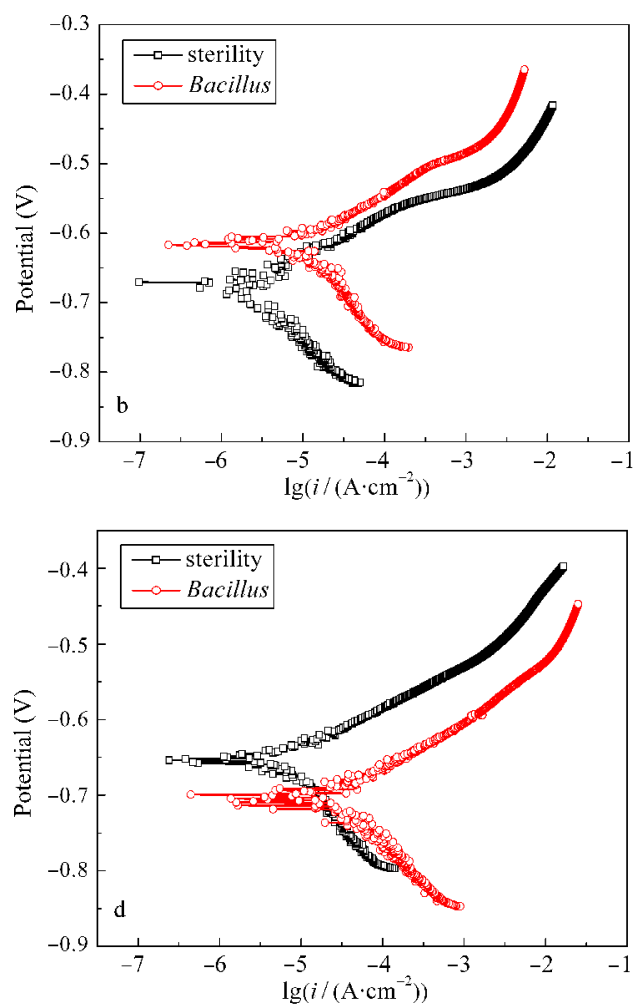

图 $6 \mathrm{~A} 3$ 钢电极浸泡在无菌溶液和芽孢杆菌菌液中不同天数的极化曲线

Fig.6 Polarization curves of steel A3 electrodes exposed in sterile and Bacillus solutions for different days t/d: (a) 2 , (b) 7, (c) 14, ,d) 21 
白培养基体系, 自腐蚀电流密度也增大至与空白培 养体系相当, 第 $21 \mathrm{~d}$ 时, 含菌体系的自腐蚀电位远 低于空白培养基体系, 腐蚀电流也相对较大, 此时, 腐蚀速率达到浸泡阶段的最大值.

\section{5 腐蚀产物形貌及失重分析}

将 A3 钢片浸泡于不同体系 $21 \mathrm{~d}$ 后取出, 可以 观察到所有试样表面都有大量腐蚀产物存在. 通过 扫描电镜对各体系的腐蚀产物进行微区观察, 结果 如图 7 所示. 图 7a 为空白培养基中试样表面腐蚀产 物的形貌图, 可以看出, 腐蚀产物以层为单位逐渐积 累, 膜层结构较为致密、光滑, 但是层与层连结并不紧 密, 发生局部产物剥落的现象. 在芽孢杆菌菌液中浸 泡的试样表面腐蚀产物层(图 7b)出现蜂窝状结构, 与空白培养基的试样相比, 虽然具有较好的均匀性, 但是膜层致密性很差, 大量的孔洞成为 $\mathrm{O}_{2}$ 分子穿过 膜层与基体进行反应的通道, 在试样表面形成了氧 浓度、离子浓度梯度, 进一步促进了腐蚀进程.

图 8 为不同体系中 $\mathrm{A} 3$ 钢失重速率随时间的变 化曲线. 从图中可以看出, 在空白培养基中浸泡试样 的平均失重速率从 0-7 d 一直增大, 7-14 d 减小, 14-21 d 又开始增大. 形成这一规律的原因主要是
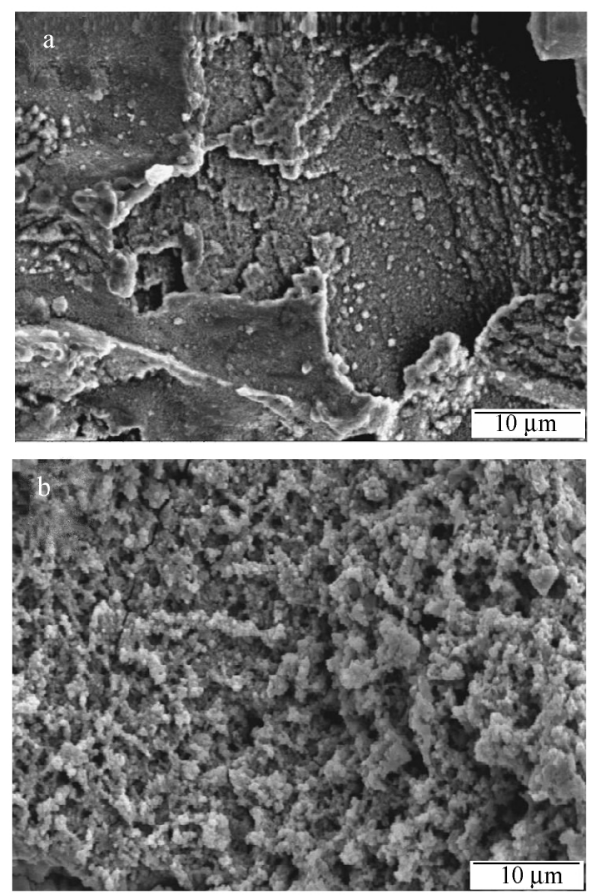

图 $7 \mathrm{~A} 3$ 钢浸泡在空白培养基(a)和芽孢杆菌菌液(b)中 $21 \mathrm{~d}$ 后的腐蚀产物形貌扫描电镜图

Fig.7 Scanning electronic microscopy (SEM) images of the corrosion products of steel A3 exposed in sterile solution (a) and Bacillus solution (b) for 21 days

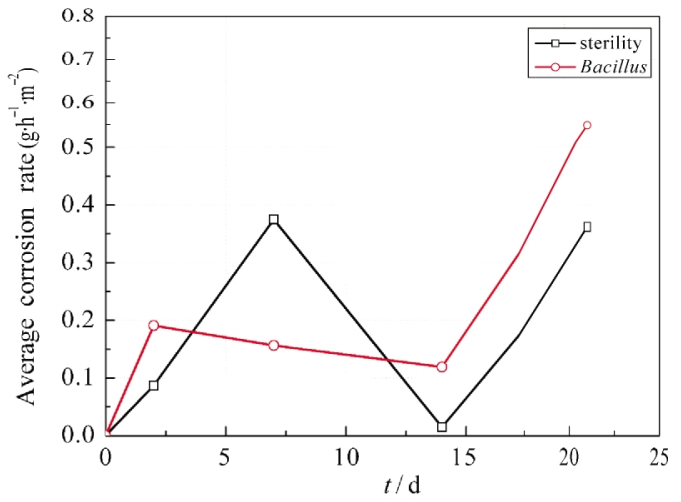

图 $8 \mathrm{~A} 3$ 钢在不同体系中腐蚀失重速率随时间的变化曲线

Fig.8 Curves of corrosion mass loss rates of steel A3 in different systems with immersion time

因为: 第 0-2 d, 金属裸露于离子溶液中, 活泼金属 直接作用于试样表面, 此时金属溶解的速率很大; 第 2-7 d, 金属表面逐渐有腐蚀产物堆积, 然而此时 的产物不足以覆盖整个试样表面, 这种不同区域腐 蚀环境的差异使得腐蚀进一步加速; 第 7-14 d, 产 物累积到一定程度, 在试样表面与溶液间建立起较 为完整的屏障, 阻碍了腐蚀进程; 第 14-21 d, 表面 膜层部分局域发生剥落, 膜层厚度的不同引起腐蚀 速率又开始增加. 在芽孢杆菌菌液中浸泡的试样表 现出完全不同的腐蚀速率变化规律: 第 0-2 d 腐蚀 失重速率增大, 之后持续减小到 $14 \mathrm{~d}$, 第 14-21 d 剧 烈增加. 形成这一规律的原因主要是由于: 第 0-2 d, 芽孢杆菌在试样表面吸附并开始分泌胞外聚合物, 生物膜逐渐成形, 可是此时无论是生物膜的厚度、均 匀度还是致密度都非常低, 使得金属的溶解速率依 然很大; 第 2-7 d, 芽孢杆菌中试样表面的生物膜变 得更加均匀、致密, 生物膜的存在成为腐蚀产物扩散 的屏障, 同时降低了电极表面的氧浓度, 由此抑制了 金属溶解的进程 ${ }^{[19]}$, 此外, 可能芽孢杆菌的代谢产物 抑制了碳钢的腐蚀; 第 7-14 d, 生物膜继续成长, 同 时试样表面还有大量腐蚀产物的累积, 两个膜层相 辅相承, 使金属的溶解进一步减缓; 第 14-21 d, 芽 孢杆菌逐渐死亡, 用来填补细菌间及产物间空隙的 胞外聚合物逐渐溶解, 此时试样表面出现大面积的 孔洞, 腐蚀速率急剧增大. 比较两个体系的腐蚀速率 曲线, 可以看出, 在 0-2 d 期间, 与空白培养基中的 试样相比, 芽狍杆菌中浸泡的试样由于生物膜不完 整反而形成了微区差异, 因此这段时间芽狍杆菌中 试样的腐蚀速率高于无菌体系; 当生物膜完整、致 密化后, 含菌体系的腐蚀速率小于无菌体系, 直至 
$12 \mathrm{~d}$, 含菌体系的腐蚀速率又高于无菌体系, 这是由 于生物膜对基体的保护作用小于腐蚀产物的保护作 用; 第 14-21 d, 含菌体系的试样由于膜层缺少 EPS 的有效填补, 较空白培养基更为疏松多孔, 腐蚀更为 严重。

\section{3 结 论}

（1）芽孢杆菌的存在会在 A3 钢表面形成致密 的生物膜, 该膜层在金属与溶液间起到很好的机械 阻隔作用, 抑制腐蚀过程.

(2) 电化学交流阻抗测试结果表明, 芽孢杆菌中 浸泡试样的时间常数在第 7 和 $14 \mathrm{~d}$ 有 3 个, 即电极 表面有生物膜和腐蚀产物膜两个膜层, 在第 $21 \mathrm{~d}$ 时 间常数只有 2 个, 即电极表面只有一个残余生物膜 和腐蚀产物膜杂合而成的复杂膜层.

(3) 微生物的活性对生物膜在电极表面所起保 护作用有决定性的影响, 当微生物活性下降, 生物膜 的保护能力也会大幅降低.

\section{References}

1 Jiang, B.; Du, C. W.; Li, X. G.; Gong, A. J. Corrosion \& Potection in Petrochemical Industry, 2008, 25(4): 1 [蒋 波, 杜翠薇, 李晓刚, 弓爱君. 石油化工腐蚀与防护, 2008, 25(4): 1]

2 Yuan, B.; Li, G. C.; Chen, Y. Materials Protection, 2005, 38(4): 38 [袁 斌, 刘贵昌, 陈 野. 材料保护, 2005, 38(4): 38]

3 Miranda, E.; Bethencourt, M.; Botana, F. J. Corrosion Science, 2006, 48: 2417

4 Liu, J. H.; Liang, X.; Li, S. M. Journal of Rare Earths, 2007, 25 (5): 609

5 Zheng, Q.; Li, J.; Du, Y. L.; Yang, C. Y. Journal of Chinese Society for Corrosion and Protection, 2008, 28(1): 38 [郑 强, 李 进, 杜一立, 杨春雨. 中国腐蚀与防护学报, 2008, 28(1): 38]

6 Fang, S. J.; Liu, Y. H.; Wang, Q.; Yu, S. R.; Song, Y. L. Journal of South China University of Technology, 2008, 36(7): 92 [方世杰,
刘耀辉, 王 强, 于思荣, 宋雨来. 华南理工大学学报, 2008, 36 (7): 92]

7 Ma, J.; Liu, H.; Shi, Y.; He, J.; Yao, S. K. Chinese Journal of Applied Chemistry, 2008, 25(7): 815 [马 洁, 刘 辉, 石 英, 贺 晶, 姚思科. 应用化学, 2008, 25(7): 815]

8 Rajasekar, A.; Babu, T. G.; Pandian, S. K.; Maruthamuthu, S.; Palaniswamy, N.; Rajendran, A. Corrosion Science, 2007, 49: 2694

9 Breed, R. E.; Gibbons, N. E. Bergey's manual of determinative bacteriology. 8th ed. in Chinese. Beijing: Science Press, 1984: 631-638 [R. E. 布坎南, N. E. 吉本斯. 伯杰细菌鉴定手册. 第八 版中文版. 北京: 科学出版社, 1984: 631-638]

10 Hui, M.; Dou, L. N.; Tian, Q.; Hou, Y. C. Journal of Anhui Agricultural Science, 2008, 36(27): 11623 [惠 明, 窦丽娜, 田 青, 侯银臣. 安徽农业科学, 2008, 36(27): 11623]

11 Li, Q. B.; Zhu, S. M.; Xu, F.; Wang, X. Environmental Science \& Technology, 2007, 30(5): 89 [李青涁, 褚松茂, 徐 伏, 王 香. 环境科学与技术, 2007, 30(5): 89]

12 Liu, G. H.; Lin, N. Q.; Lin, Y. Z.; Liu, B. Fujian Journal of Agricultural Sciences, 2008, 23(1): 92 [刘国红, 林乃铨, 林营 志, 刘 波. 福建农业学报, 2008, 23(1): 92]

13 Juzeliūnas, E.; Ramanauskas, R.; Lugauskas, A.; Leinartas, K.; Samulevčienè, M.; Sudavicius, A. Electrochimica Acta, 2006, 51: 6085

14 Wang, W.; Wang, J.; Xu, H. B.; Li, X. B. Corrosion Science and Protection Technology, 2007, 19(1): 38 [王＼cjkstart伟, 王 佳, 徐海 波, 李相波. 腐蚀科学与防护技术, 2007, 19(1): 38]

15 Masaaki, M. Journal of Bioscience and Bioengineering, 2006, 101 (1): 1

16 Juzeliūnas, E.; Ramanauskas, R.; Lugauskas, A.; Samuleverienè, M.; Leinartas, K. Electrochemistry Communications, 2005, 7: 305

17 Zuo, R.; Kus, E.; Mansfeld, F.; Wood, T. K. Corrosion Science, 2005, 47: 279

18 Sheng, X. X.; Ting, Y. P.; Pehkone, S. O. Corrosion Science, 2007, 49: 2159

19 Chongdar, S.; Gunasekaran, G.; Kumar, P. Electrochimica Acta, 2005, 50: 4655 